\title{
Serial C-reactive Protein Monitoring in Prosthetic Joint Infection: A Powerful Predictor or Potentially Pointless?
}

\author{
Rafia Ghani $^{1}$, Jonathan Hutt ${ }^{2}$, Philip Mitchell ${ }^{2}$, Luke Granger ${ }^{2}$, Nemandra A. Sandiford ${ }^{2}$ \\ 1. Orthopaedics, Russells Hall Hospital, Dudley, GBR 2. Orthopaedics, St. George's University Hospital, London, GBR
}

Corresponding author: Rafia Ghani, rafia.ghani@nhs.net

\section{Abstract \\ Background}

Serum C-reactive protein (CRP) is an important test in the initial diagnosis of prosthetic joint infection (PJI). There is no widely accepted algorithm for the resolution of PJI. Surgeons have traditionally used CRP to determine if the infection has resolved. However, this practice is not currently supported by significant data.

\section{Methods}

A retrospective analysis of our departmental arthroplasty database was conducted to determine mean values of CRP pre and postoperatively for PJI treated with the debridement, antibiotics and implant retention (DAIR) procedure, single-stage revision and two-stage revision. Receiver operating characteristic (ROC) curves were calculated to determine the sensitivity and specificity of CRP testing in diagnosing persistent infection.

\section{Results}

Of the 121 patients who had undergone treatment (75 hip replacements and 48 knee replacements), there were 26 cases of persistent infection. There was no statistical significance in the mean CRP values between successful and unsuccessful treatment groups. The areas under ROCs (AUCs) for CRP values predicting outcomes ranged from 0.46 to 0.73 .

\section{Conclusion}

Our study does not support the use of serial CRP monitoring as an indicator of the successful eradication of PJI.

\section{Received 02/06/2020 Review began 02/09/2020 Review ended 02/10/2020 Published 02/12/2020 \\ (c) Copyright 2020 Ghani et al. This is an open access article distributed under the terms of the Creative Commons Attribution License CC-BY 4.0., which permits unrestricted use, distribution, and reproduction in any medium, provided the original author and source are credited.}

Categories: Infectious Disease, Orthopedics, Healthcare Technology

Keywords: arthroplasty, orthopaedics, infection, prosthetic, prosthetic joint, revision, blood testing, inflammatory

\section{Introduction}

Prosthetic joint infection (PJI) is a serious complication of total joint arthroplasty of the hip and knee. It is estimated that $1-2.5 \%$ of patients undergoing primary total joint replacement require treatment for PJI [1]. There are well-established evidence-based algorithms in place for the initial diagnosis of PJI with considerable evidence to support the use of serum inflammatory markers in diagnosis [2]. Following the diagnosis of PJI, the main treatment modalities are debridement, antibiotics and implant retention (DAIR), single-stage revision or two-stage revision. Two-stage revision remains the gold standard of treatment for PJI [3]. In all treatment modalities, there is no widely accepted algorithm to determine infection resolution and the success of treatment. Many surgeons use serial serum C-reactive protein (CRP, an inflammatory marker) monitoring to determine response to treatment. However, there is no reliable evidence yet to suggest that low or decreasing CRP values indicate the elimination of infection.

Current evidence suggests that serial CRP monitoring cannot reliably determine infection control in twostage revision; however, the role of CRP in assessing the success in DAIR and single-stage revision procedures remains unclear. Ghanem et al. studied 109 patients who had undergone two-stage revision for infected knee replacements from 1999 to 2006. They analysed the effectiveness of CRP as a test in determining the eradication of infection by using the area under a receiver operator characteristic (ROC) curve (AUC). They found the AUC for CRP to be 0.55 , which was not statistically significant. The study concluded that CRP often does not normalise even when the infection is eradicated [4]. This conclusion was supported by Shukla et al. who retrospectively reviewed serologies of 76 infected total knee arthroplasty (TKA) patients who were treated with a two-stage exchange [5]. More recently, Bejon et al. came to a similar conclusion after analysing a dataset of 151 total joint arthroplasty patients ( 71 hip, 76 knee and four elbow 


\section{Cureus}

revisions) who had undergone two-stage revision for PJI. They also analysed a dataset of 109 patients who had undergone DAIR (51 hip replacement, 50 knee replacements and eight other joints). They found that CRP had an AUC of 0.65 for predicting failure of DAIR at one year and concluded that CRP testing in this subgroup was of marginal usefulness [6].

Our aim was to examine the usefulness of CRP testing in determining whether a PJI has been treated successfully.

\section{Materials And Methods}

Three clinical datasets were retrospectively gathered from patients with PJI managed in a single tertiary referral centre specialising in treating PJI between April 2011 and March 2017. All cases treated with DAIR, single or two-stage revisions for PJI were included. Infection was diagnosed according to the Musculoskeletal Infection Society (MSIS) criteria [2]. All patients had been treated by the same surgical team. In all cases, patients had been treated with antibiotics for at least six weeks postoperatively according to organism sensitivity.

CRP results were collected preoperatively and at weeks one, three and six postoperatively. These results were collected from the electronic pathology reporting system. Clinical notes were reviewed for each patient to determine the organism responsible for PJI and to identify cases with persistent infection after treatment. The condition of persistent infection was defined based on the following criteria: (i) requiring further surgery to eradicate infection, (ii) presence of fever, rigors or purulent drainage postoperatively or (iii) chronic joint pain and swelling lasting 1-2 years postoperatively.

All data analysis was performed using Statistical Product and Service Solutions (SPSS) software version 25 (IBM, Armonk, NY) with significance set at a $=0.05$. Mean values for CRP were calculated preoperatively and at weeks one, three and six postoperatively for DAIR, single-stage revision and two-stage revision respectively. Receiver operating characteristic (ROC) curves were calculated from mean CRP values. This allowed us to examine the area under the curve (AUC). The area under the curve determines how well a test separates the group being tested into those with and without the disease in question [7]. The traditional academic point system is a guide for determining the accuracy of a diagnostic test (Table 1 ).

\begin{tabular}{|l|l|}
\hline AUC value & Accuracy of diagnostic test \\
\hline $.90-1$ & Excellent \\
$.80-.90$ & Good \\
$.70-.80$ & Fair \\
$.60-.70$ & Poor \\
$.50-.60$ & Fail \\
\hline
\end{tabular}

\section{TABLE 1: Traditional academic point system for AUC test values}

AUC: area under receiver operating characteristic curve

There was no research-related contact with patients and all data was anonymised. Informed consent was waived for our study.

\section{Results}

A total of 121 (hip 73, knee 48) cases were identified. Of these cases, 68 (hip 43, knee 25) had been treated with single-stage revision, 24 (hip 15, knee 9) with two-stage revision and 29 (hip 15, knee 14) with DAIR. The average age of the patient was 68 (range: 27-90) with 61 male patients and 60 female patients. PJI was found to be eradicated in 95 of the 121 patients (79\%) in the cohort. There were 11 cases of persistent infection in 68 patients (16\%) treated with single-stage revision. There were nine cases of persistent infection in 24 patients (38\%) treated with two-stage revision. There were six cases of persistent infection in 29 patients (21\%) treated with DAIR.

There were 444 CRP results collected for 121 patients. There were 241 CRP results for 61 patients in the single-stage revision cohort (comprising 43 hip replacements and 25 knee replacements). There were 92 CRP results collected for 24 patients in the two-stage revision cohort (comprising 15 hip replacement and nine knee replacements). There were $111 \mathrm{CRP}$ results collected for 29 patients in the DAIR cohort (comprising 15 hip replacements and 14 knee replacements). 


\section{Cureus}

\section{Single-stage revision}

The mean preoperative CRP of the single-stage revision cohort was 62 [95\% confidence interval (CI): 42-83]. One week postoperatively, the mean CRP of the cohort was 50 (95\% CI: 40-59). At week three postoperatively, the mean was 43 (95\% CI: 24-61). At week six postoperatively, the mean was 23.66 (95\% CI: 15-33) (Table 2). There was no statistically significant difference between the mean CRP for patients who remained persistently infected and those who remained uninfected. ROC curves were produced using the mean CRP values of the cohort. The AUC values for weeks one and three were 0.471 and 0.421 respectively, indicating that testing at this time led to poor results in predicting reinfection (Figure 1) (Table 3). The AUC at week 6 was 0.733 , indicating that this CRP testing was moderately useful; however, this test was not statistically significant (p: 0.67 ).

\begin{tabular}{|c|c|c|c|}
\hline Inflammatory marker & Mean & $95 \%$ confidence interval & Standard deviation \\
\hline Preoperative CRP & 62.26 & $41.95-82.56$ & 65.99 \\
\hline Week-1 CRP & 49.75 & $40.35-59.16$ & 30.56 \\
\hline Week-3 CRP & 42.79 & $24.42-61.16$ & 59.70 \\
\hline Week-6 CRP & 23.66 & $14.75-32.57$ & 28.95 \\
\hline
\end{tabular}

\section{TABLE 2: Mean CRP values in single-stage revision cohort}

CRP: C-reactive protein
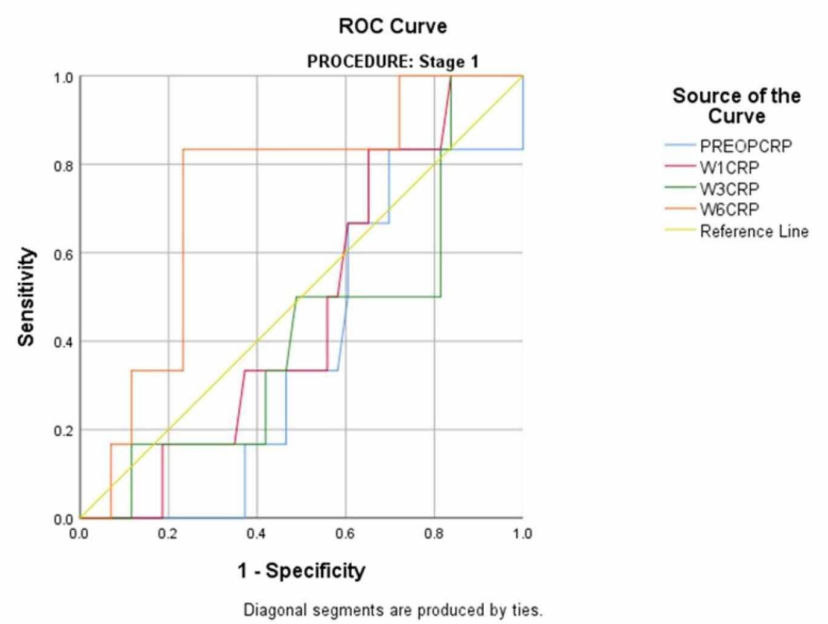

FIGURE 1: receiver operating characteristic curve for single-stage revision

ROC: receiver operating characteristic

PREOPCRP: preoperative C-reactive protein

W1CRP: week-1 C-reactive protein

W3CRP: week-3 C-reactive protein

W6CRP: week-6 C-reactive protein 


\section{Cureus}

\begin{tabular}{|c|c|c|c|c|c|}
\hline \multirow{2}{*}{ Test result variable(s) $^{\mathrm{a}}$} & \multirow{2}{*}{ Area } & \multirow{2}{*}{ Std. error ${ }^{b}$} & \multirow{2}{*}{ Asymptotic significance ${ }^{c}$} & \multicolumn{2}{|c|}{ Asymptotic $95 \%$ confidence interva } \\
\hline & & & & Lower bound & Upper bound \\
\hline Preoperative CRP & .378 & .099 & .337 & .184 & .572 \\
\hline Week-1 CRP & .471 & .102 & .819 & .270 & 671 \\
\hline Week-3 CRP & .421 & .120 & .532 & .185 & .657 \\
\hline Week-6 CRP & .733 & .100 & .067 & .537 & .928 \\
\hline \multicolumn{6}{|l|}{ a. Procedure = stage 1} \\
\hline \multicolumn{6}{|c|}{ b. Under the nonparametric assumption } \\
\hline \multicolumn{6}{|c|}{ c. Null hypothesis: true area $=0.5$} \\
\hline
\end{tabular}

TABLE 3: Area under the curve values for single-stage revision

CRP: C-reactive protein

\section{Two-stage revision}

The mean preoperative CRP of the two-stage revision cohort was 63 (95\% CI: 39-88). One week postoperatively, the mean CRP of the cohort was 60 (95\% CI: 29-91). At week three postoperatively, the mean was 36 (95\% CI: 18-53). At week six postoperatively, the mean was 23 (95\% CI: 13-33) (Table 4). There was no statistically significant difference between the mean CRP for patients who remained persistently infected and those who remained uninfected. ROC curves were produced using the mean CRP values of the cohort. The AUC values for CRP testing in this cohort at all times were $<0.6$, indicating that the test was not useful (Figure 2) (Table 5).

\begin{tabular}{|c|c|c|c|}
\hline Inflammatory marker & Mean & $95 \%$ confidence interval & Standard deviation \\
\hline Preoperative CRP & 62.52 & 39.26-85.79 & 46.78 \\
\hline Week-1 CRP & 59.90 & $28.79-91.01$ & 62.56 \\
\hline Week-3 CRP & 35.67 & $18.25-53.08$ & 35.03 \\
\hline Week-6 CRP & 22.98 & $12.60-33.35$ & 20.86 \\
\hline
\end{tabular}

TABLE 4: Mean CRP values in two-stage revision cohort

CRP: C-reactive protein
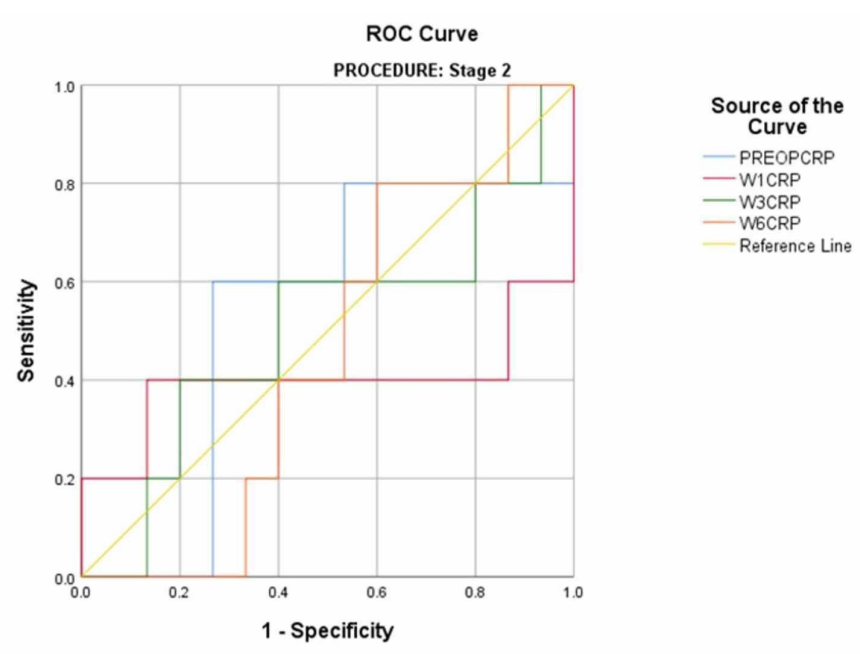


\section{Cureus}

FIGURE 2: receiver operating characteristic curve for second-stage revision

ROC: receiver operating characteristic

PREOPCRP: preoperative C-reactive protein

W1CRP: week-1 C-reactive protein

W3CRP: week-3 C-reactive protein

W6CRP: week-6 C-reactive protein

\begin{tabular}{|c|c|c|c|c|c|}
\hline \multirow{2}{*}{ Test result variable(s) ${ }^{a}$} & \multirow{2}{*}{ Area } & \multirow{2}{*}{ Std. error ${ }^{b}$} & \multirow{2}{*}{ Asymptotic significance ${ }^{c}$} & \multicolumn{2}{|c|}{ Asymptotic $95 \%$ confidence interval } \\
\hline & & & & Lower bound & Upper bound \\
\hline Preoperative CRP & .533 & 156 & .827 & .227 & .840 \\
\hline Week-1 CRP & .400 & .200 & .513 & .008 & .792 \\
\hline Week-3 CRP & .507 & .162 & .965 & 188 & .825 \\
\hline Week-6 CRP & .453 & .132 & .760 & .194 & .713 \\
\hline
\end{tabular}

a. Procedure $=$ stage 2

b. Under the nonparametric assumption

c. Null hypothesis: true area $=0.5$

TABLE 5: Area under the curve values for second-stage revision

CRP: C-reactive protein

\section{Debridement, antibiotics and implant retention}

The mean pre-operative CRP of the DAIR cohort was 131 (95\% CI: 86-177). One week postoperatively, the mean CRP of the cohort was 72 (95\% CI: 51-94). At week three postoperatively, the mean was 59 (95\% CI: 3188). At week six post-operatively, the mean was 56 (95\% CI: 21-91) (Table 6). There was no statistically significant difference between the mean CRP for patients who remained persistently infected and those who remained uninfected. ROC curves were produced using the mean CRP values of the cohort. The AUC values for CRP testing in this cohort at all times were $<0.6$, indicating that the test was not useful (Figure 3) (Table 7).

\begin{tabular}{|c|c|c|c|}
\hline Inflammatory marker & Mean & $95 \%$ confidence interval & Standard deviation \\
\hline Preoperative CRP & 131.63 & $86.11-177.15$ & 107.80 \\
\hline Week-1 CRP & 72.16 & 50.54-93.79 & 51.21 \\
\hline Week-3 CRP & 59.49 & $30.64-88.33$ & 68.31 \\
\hline Week-6 CRP & 56.36 & 22.13-90.60 & 81.10 \\
\hline
\end{tabular}

\section{TABLE 6: Mean CRP values in DAIR cohort}

DAIR: debridement, antibiotics and implant retention

CRP: C-reactive protein 


\section{Cureus}

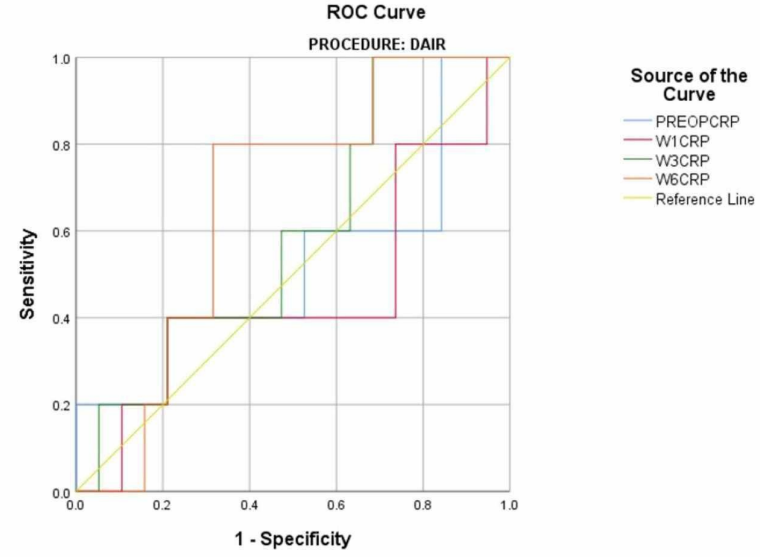

\section{FIGURE 3: Receiver operator curve for DAIR}

ROC: receiver operating characteristic

DAIR: debridement, antibiotics and implant retention

PREOPCRP: preoperative C-reactive protein

W1CRP: week-1 C-reactive protein

W3CRP: week-3 C-reactive protein

W6CRP: week-6 C-reactive protein

\begin{tabular}{|c|c|c|c|c|c|}
\hline \multirow{2}{*}{ Test result variable(s) $^{a}$} & \multirow{2}{*}{ Area } & \multirow{2}{*}{ Std. error ${ }^{b}$} & \multirow{2}{*}{ Asymptotic significance $^{c}$} & \multicolumn{2}{|c|}{ Asymptotic $95 \%$ confidence interval } \\
\hline & & & & Lower bound & Upper bound \\
\hline Preoperative CRP & .516 & 164 & .915 & 195 & .837 \\
\hline Week-1 CRP & .453 & 162 & .749 & 136 & .769 \\
\hline Week-3 CRP & .589 & .135 & .546 & .325 & .854 \\
\hline Week-6 CRP & .663 & .120 & .271 & .429 & .897 \\
\hline
\end{tabular}

a. Procedure $=$ DAIR

b. Under the nonparametric assumption

c. Null hypothesis: true area $=0.5$

\section{TABLE 7: Area under the curve values for DAIR}

DAIR: debridement, antibiotics and implant retention

CRP: C-reactive protein

\section{Discussion}

Based on our results, we could not recommend CRP as a test for determining the eradication of PJI. Our analysis generated low AUC values for all treatment modalities, indicating poor sensitivity and specificity of the test. This could potentially reflect the limited power of the study. However, it is more likely that the wide scatter of readings could be contributing to this. Treatment failure after DAIR, single-stage and two-stage revision was not associated with a high CRP at any measured time point. There was no statistical difference in mean CRP between patients with treatment failure and those who had successful treatment with any treatment modality. 
PJI is a significant problem and carries a high morbidity rate for the patients. it is also becoming increasingly expensive to manage with a projected spend of US\$ 1.62 billion in the US alone [8]. Research from Europe shows that the presence of infection triples the cost of a primary joint arthroplasty [9]. There have been many advances in surgical technique and preoperative diagnosis; however, there remains less clarity and consensus on the optimal criteria to assess response to treatment [2]. We feel it is essential to have a prognostic algorithm that allows for the use of inexpensive and readily available tests. Our research supports the current consensus that CRP in isolation is not useful in determining the eradication of infection. Recent studies have shown that interleukin-6 (IL-6) has a higher specificity for detecting the presence of infection; however, this test remains relatively expensive and inaccessible to the average surgeon [10,11].

We feel that more work needs to be done in reaching a consensus on how to monitor PJI treatment. A potential topic of further study would be to examine the usefulness of CRP in conjunction with other inflammatory markers such as white cell count and erythrocyte sedimentation rate.

\section{Conclusions}

Our study found that serial CRP testing was not a reliable test for determining the eradication of PJI in cases treated with single-stage revision, two-stage revision or DAIR. We feel that more work needs to be done to establish a widely accepted and reliable algorithm to determine the resolution of the infection.

\section{Additional Information \\ Disclosures}

Human subjects: Consent was obtained by all participants in this study. Animal subjects: All authors have confirmed that this study did not involve animal subjects or tissue. Conflicts of interest: In compliance with the ICMJE uniform disclosure form, all authors declare the following: Payment/services info: All authors have declared that no financial support was received from any organization for the submitted work. Financial relationships: All authors have declared that they have no financial relationships at present or within the previous three years with any organizations that might have an interest in the submitted work. Other relationships: All authors have declared that there are no other relationships or activities that could appear to have influenced the submitted work.

\section{References}

1. Tande AJ, Patel R: Prosthetic joint infection. Clin Microbiol Rev. 2014, 27:302-345. 10.1128/CMR.00111-13

2. Parvizi J, Tan TL, Goswami K, Higuera C, Della Valle C, Chen AF, Shohat N: The 2018 definition of periprosthetic hip and knee infection: an evidence-based and validated criteria. J Arthroplasty. 2018, 33:1309-1314. 10.1016/j.arth.2018.02.078

3. Charette RS, Melnic CM: Two-stage revision arthroplasty for the treatment of prosthetic joint infection . Curr Rev Musculoskelet Med. 2018, 11:332-340. 10.1007/s12178-018-9495-y

4. Ghanem E, Azzam K, Seeley M, Joshi A, Parvizi J: Staged revision for knee arthroplasty infection: what is the role of serologic tests before reimplantation?. Clin Orthop Relat Res. 2009, 467:1699-1705. 10.1007/s11999009-0742-9

5. Shukla SK, Ward JP, Jacofsky MC, Sporer SM, Paprosky WG, Della Valle CJ: Perioperative testing for persistent sepsis following resection arthroplasty of the hip for periprosthetic infection. J Arthroplasty. 2010, 25:87-91. 10.1016/j.arth.2010.05.006

6. Bejon P, Byren I, Atkins BL, et al.: Serial measurement of the C-reactive protein is a poor predictor of treatment outcome in prosthetic joint infection. J Antimicrob Chemother. 2011, 66:1590-1593. 10.1093/jac/dkr182

7. The area under an ROC curve . (2020). Accessed: February 5, 2020: http://gim.unmc.edu/dxtests/ROC3.htm.

8. Haddad FS, Ngu A, Negus JJ: Prosthetic joint infections and cost analysis?. Adv Exp Med Biol. 2017, 971:93100. 10.1007/5584_2016_155

9. Puhto T, Puhto AP, Vielma M, Syrjälä H: Infection triples the cost of a primary joint arthroplasty . Infect Dis (Lond). 2019, 51:348-355. 10.1080/23744235.2019.1572219

10. Lenski M, Scherer MA: Synovial IL-6 as inflammatory marker in periprosthetic joint infections . J Arthroplasty. 2014, 29:1105-1109. 10.1016/j.arth.2014.01.014

11. Xie K, Dai K, Qu X, Yan M: Serum and synovial fluid interleukin-6 for the diagnosis of periprosthetic joint infection. Sci Rep. 2017, 7:1496. Accessed: February 12, 2020: https://www.ncbi.nlm.nih.gov/pubmed/28473693. 10.1038/s41598-017-01713-4 